\title{
Combined Bacterial and Herpes Simplex Virus Keratitis following Small-Incision Lenticule Extraction for the Correction of Myopia
}

\author{
Arturo Ramirez-Miranda Simran Mangwani-Mordani \\ Natalia Quiroz-Casian Valeria Oliva-Bienzobas Jesus Cabral-Macias \\ Alejandro Navas Enrique O. Graue-Hernandez \\ Department of Cornea and Refractive Surgery, Instituto de Oftalmología, Conde de \\ Valenciana, Mexico City, Mexico
}

Keywords

Small-incision lenticule extraction · Keratitis · Herpes simplex virus

\begin{abstract}
A 28-year-old male presented unilateral visual loss, intense ocular pain, redness and intraocular hypertension in his right eye 2 days after undergoing small-incision lenticule extraction (SMILE) in both eyes. Initial examination of the affected eye revealed the presence of white infiltrates within the corneal interface, as well as a central epithelial defect. The patient was diagnosed with infectious keratitis, posteriorly the eye was irrigated with balanced saline solution and treatment was initiated with hourly moxifloxacin $0.5 \%$. Since this approach failed to resolve symptoms, a sample from the interface was obtained for PCR assay, which revealed the presence of herpes simplex virus DNA, confirming the cause of the infection. The patient was prescribed a regimen of oral acyclovir, topical ganciclovir and prednisolone. Clinical improvement following resolution of the epithelial defect was observed. Although rare, herpetic keratitis following SMILE is best managed via early diagnosis and initiation of appropriate anti-herpetic treatment.
\end{abstract}

\section{Introduction}

Small-incision lenticule extraction (SMILE) is a novel keratorefractive procedure that has been shown to be a safe and an effective alternative to laser-assisted in situ keratomileusis (LASIK) for correction of myopia and myopic astigmatism [1]. In SMILE, an intrastromal 
lenticule is created using a femtosecond laser; the lenticule is then extracted manually via a small peripheral incision in the corneal surface [2]. Although the procedure is generally safe some complications have been reported, including suction loss, black spots, and lenticule misdissection [3]. The overall incidence of infectious keratitis following SMILE is unknown [4]; nonetheless, postoperative infections have been relatively rare for most refractive procedures $[5,6]$. In this article, we report the first documented case of unilateral herpetic keratitis (ocular herpes simplex virus [HSV]) following SMILE for the correction of myopia.

\section{Case Report}

A 28-year-old male underwent simultaneous bilateral SMILE for the correction of myopia at a private clinic; he presented to our clinic 2 days later with unilateral visual loss and intense ocular pain in his right eye. His medical history indicated that he was immunocompetent prior to surgery, with no precedent of HSV keratitis and no evident risk factors for infections. He was not a contact lens wearer.

At presentation, the patient's uncorrected distance visual acuity (UDVA) was reduced to counting fingers at $30 \mathrm{~cm}$ in the right eye and 20/20 with the unaffected left eye. Ocular slitlamp examination of the right eye revealed several white infiltrates within the interface, a central epithelial defect, keratic precipitates on the endothelium, 2+ cells in the anterior chamber (shown in Fig. 1a-c), and intraocular hypertension. Lens and fundus details could
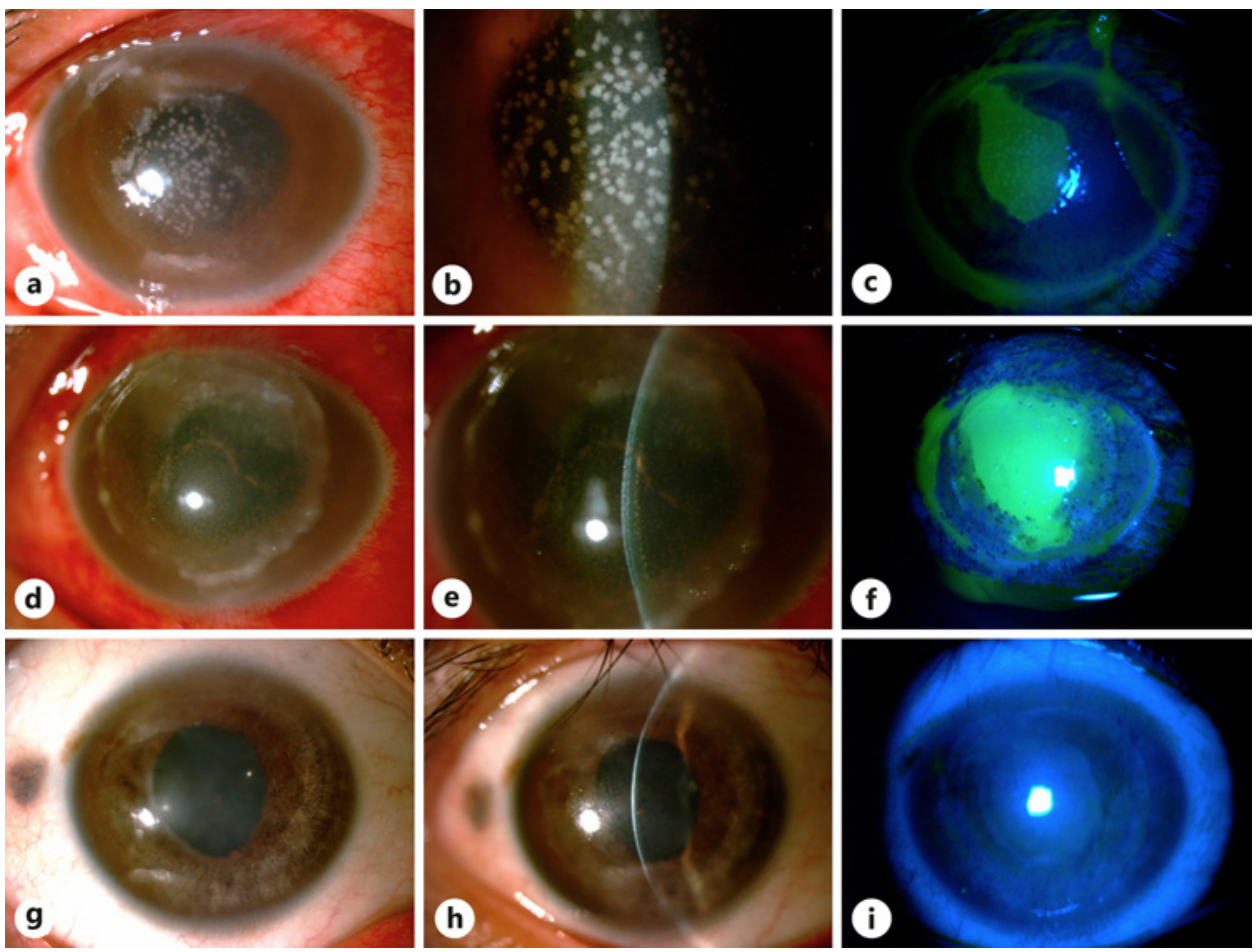

Fig. 1. Clinical progression of herpetic keratitis in post-SMILE patient. a-c Clinical images of the patient's right eye at day 2 postoperatively. Multiple, stromal, white infiltrates were evident within the interface area, and there was a central epithelial defect related to the surgical incision. $\mathbf{d}-\mathbf{f}$ Clinical evolution following rinsing of the stromal pocket with balanced salt solution and the administration of moxifloxacin eye drops. Endothelial deposits were evident and a peripheral ring-shape infiltrate appeared. In addition, the epithelial defect was larger than previously observed. g-i Clinical images at 3-month follow-up showing absolute absence of corneal infiltrates and edema. A complete reepithelization was achieved. SMILE, small-incision lenticule extraction. 
not be assessed due to hazy media. An initial diagnosis of unilateral infectious keratitis was made, and the patient was moved to the clinic's operating room, where his right eye was irrigated with balanced saline solution and topical moxifloxacin was administered via the stromal pocket (as seen in Fig. 1d-f).

Simultaneously, corneal scrapes from the stromal pocket were collected for microscopic examination, inoculation of culture media (blood agar, chocolate agar, Sabouraud dextrose agar, and Mannitol salt agar), gram staining, and PCR assay. The patient initiated hourly moxifloxacin $0.5 \%$, tropicamide/phenylephrine, topical brimonidine with timolol and dorzolamide, preservative-free eye drops, oral doxycycline, and oral vitamin C. Anterior segment spectral-domain optical coherence tomography was performed daily to monitor the patient's overall ocular health.

Gram staining revealed gram-positive cocci in the right eye, and the patient's symptoms worsened despite treatment. PCR assay of corneal tissue revealed the presence of HSV DNA, suggesting unilateral stromal herpetic keratitis.

The patient was prescribed an additional regimen of oral acyclovir $(400 \mathrm{mg}, 5$ times daily) and topical $0.15 \%$ ganciclovir gel; he experienced clinical improvement within 2 weeks, as the epithelial defect healed. The patient was then prescribed topical steroids (prednisolone acetate $1.0 \%, \mathrm{Q} 2 \mathrm{H}$ ), which resulted in a reduction of corneal infiltrates in the affected eye. After 3 weeks of treatment, the infection by HSV resolved (Fig. 1g-i), although the patient's UDVA remained at counting fingers at $30 \mathrm{~cm}$. Six months later, he had UDVA of 20/400, and there was no evidence of HSV recurrence. Patient signed informed consent to publish this case end the images of his eye. The patient's initial exam findings (upon presenting to our clinic) as well as his key findings after initiation of treatment for herpetic keratitis are summarized in Table 1.

\section{Discussion}

To our knowledge, this case report describes the first incidence of postoperative herpetic keratitis in a patient undergoing the SMILE procedure. The case is particularly noteworthy, given that the patient was otherwise healthy and had no obvious risk factors for ocular HSV.

Infectious keratitis is a potentially severe and devastating complication following refractive surgery. Although studies suggest that the incidence of postoperative keratitis following refractive surgery decreased in the 2000 s, it still occurs in $0.02-0.2 \%$ of cases [6]. In most cases described in literature, it is not always possible to determine the origin of infection, yet early diagnosis and intervention with appropriate therapy usually result in full visual recovery [6]. Proper management of infectious keratitis presents a unique clinical

Table 1. Clinical findings before and after receiving treatment for herpetic keratitis

\begin{tabular}{ll}
\hline Initial findings & Findings post-treatment \\
\hline UDVA OD & \\
Counting fingers & Counting fingers \\
& $20 / 400$ (6-month follow-up) \\
\hline Ocular slit-lamp OD & \\
White infiltrates & Reduction in infiltrates \\
Central epithelial defect & Healed central epithelium defect \\
$2+$ cells in anterior chamber & No cells in anterior chamber \\
\hline
\end{tabular}

\section{Karger ${ }^{\prime}=$}


challenge; empiric therapy must be initiated prior to the availability of microbiologic results from samples of corneal tissue [7].

As with other refractive procedures affecting the cornea, it has historically been assumed that infections following SMILE occur as a result of microorganisms trapped within the stromal pocket [8]. However, only a few studies have reported infectious keratitis following SMILE. Vestergaard et al. [4], for example, reported a case involving a patient who presented presumed infectious corneal infiltrates postoperatively; however, the patient was lost to follow-up. To our knowledge, only one case of severe, bilateral infectious keratitis caused by Streptococcus pneumoniae [8] following SMILE has been reported and documented. This case was treated based on the American Society of Cataract and Refractive Surgeons recommendations for post-LASIK infections (irrigation with bactericidal povidone-iodine solution and topical corticosteroids), resulting in a favorable outcome.

To date, there is insufficient data regarding the incidence of herpetic keratitis (ocular HSV) following SMILE. Studies have suggested that the incidence of herpetic keratitis following LASIK procedure is $\leq 0.05 \%$ although it is believed that the infection in this setting has been underreported [5, 9]. In most of the documented cases, herpetic keratitis following LASIK was diagnosed on the basis of clinical examination (e.g., the presence of dendritic lesions) and confirmed by the presence of HSV DNA via PCR assay.

Although few cases have been documented, some concern still remains regarding the potential reactivation of latent herpetic eye disease following refractive surgery. This association has been previously described in animal models and reported in humans [9-11]. Several studies have demonstrated that LASIK and surface excimer laser ablation can trigger the reactivation of latent HSV; nonetheless, the exact mechanism of reactivation remains unknown [10]. In addition, some evidence suggests that reactivation may even occur in the absence of prior herpetic disease following surgery using an excimer laser, microkeratomes, and/or a femtosecond laser [5, 9, 10]. SMILE, theoretically, results in less trauma to the corneal epithelium than other refractive procedures, but could share the same triggering mechanisms for reactivation of latent HSV.

In the present case, it is difficult to ascertain whether the procedure played a role for the onset of herpetic keratitis. We believe the case highlights the importance of timely diagnosis and proper management of herpetic keratitis despite the atypical presentation, in order to maintain appropriate postoperative visual and structural results. Surgeons should remain vigilant for possible complications post-SMILE and other postoperative refractive surgery patients.

\section{Statement of Ethics}

This case report abided the tenets of the Declaration of Helsinki and obtained written informed consent to publish and use relevant images from the subject.

\section{Conflict of Interest Statement}

The authors have no conflicts of interest to declare.

\section{Funding Sources}

The authors did not receive financial support for this research.

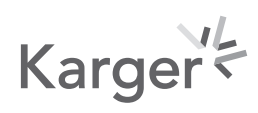


Ramirez-Miranda et al.: Infectious Keratitis after SMILE

\section{Author Contributions}

A.R.M., A.N., and E.O.G.H. conceived the original idea for this case report. A.R.M., N.Q.C., V.O.B., and J.C.M. were responsible for data acquisition including the images in Figure 1 and the findings shown in Table 1. A.R.M., S.M.M., N.Q.C., V.O.B., and J.C.M. performed data analysis. A.R.M. and S.M.M. wrote the manuscript with support from A.N. and E.O.G.H. All the authors contributed to the critical revision and final approval of the manuscript.

\section{References}

1 Lin F, Xu Y, Yang Y. Comparison of the visual results after SMILE and femtosecond laser-assisted LASIK for myopia. J Refract Surg. 2014;30(4):248-54.

2 Ivarsen A, Asp S, Hjortdal J. Safety and complications of more than 1,500 small-incision lenticule extraction procedures. Ophthalmology. 2014;121(4):822-8.

3 Ramirez-Miranda A, Ramirez-Luquin T, Navas A, Graue-Hernandez EO. Refractive lenticule extraction complications. Cornea. 2015;34(Suppl 10):S65-7.

4 Vestergaard A, Ivarsen AR, Asp S, Hjortdal JØ. Small-incision lenticule extraction for moderate to high myopia: predictability, safety, and patient satisfaction. J Cataract Refract Surg. 2012;38(11):2003-10.

5 Arora T, Sharma N, Arora S, Titiyal JS. Fulminant herpetic keratouveitis with flap necrosis following laser in situ keratomileusis: case report and review of literature. J Cataract Refract Surg. 2014;40(12):2152-6.

6 Solomon R, Donnenfeld ED, Holland EJ, Yoo SH, Daya S, Güell JL, et al. Microbial keratitis trends following refractive surgery: results of the ASCRS infectious keratitis survey and comparisons with prior ASCRS surveys of infectious keratitis following keratorefractive procedures. J Cataract Refract Surg. 2011;37(7):1343-50.

7 de Rojas V, Llovet F, Martínez M, Cobo-Soriano R, Ortega-Usobiaga J, Beltrán J, et al. Infectious keratitis in 18,651 laser surface ablation procedures. J Cataract Refract Surg. 2011;37(10):1822-31.

8 Chehaibou I, Sandali O, Ameline B, Bouheraoua N, Borderie V, Laroche L. Bilateral infectious keratitis after small-incision lenticule extraction. J Cataract Refract Surg. 2016;42(4):626-30.

9 Levy J, Lapid-Gortzak R, Klemperer I, Lifshitz T. Herpes simplex virus keratitis after laser in situ keratomileusis. J Refract Surg. 2005;21(4):400-2.

10 Lu CK, Chen KH, Lee SM, Hsu WM, Lai JY, Li YS. Herpes simplex keratitis following excimer laser application. J Refract Surg. 2006;22(5):509-11.

11 Dhaliwal DK, Romanowski EG, Yates KA, Hu D, Goldstein M, Gordon YJ. Experimental laser-assisted in situ keratomileusis induces the reactivation of latent herpes simplex virus. Am J Ophthalmol. 2001;131(4):506-7. 